Full length article

\title{
An experimental and theoretical study of duplex fcc + hcp cobalt based entropic alloys
}

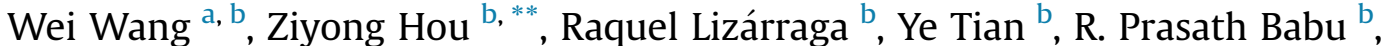 \\ Erik Holmström ${ }^{\mathrm{c}}$, Huahai Mao ${ }^{\mathrm{b}, \mathrm{d}}$, Henrik Larsson ${ }^{\mathrm{b}, \mathrm{d}, \text { * }}$ \\ a Dept Chemical Engineering, Northeast Electric Power University, Jilin, 132012, China \\ b Unit of Structures, Dept Materials Science and Engineering, KTH, SE-10044, Stockholm, Sweden \\ c Sandvik Coromant $R \&$ \&, SE-12680, Stockholm, Sweden \\ d Thermo-Calc Software, Råsundav. 18, SE-16767, Solna, Sweden
}

\section{A R T I C L E I N F O}

\section{Article history:}

Received 17 May 2019

Received in revised form 29 May 2019

Accepted 25 June 2019

Available online 27 June 2019

Keywords:

Co-Base alloys

Martensitic phase transformation

Computational thermodynamics

CALPHAD

DFT

\begin{abstract}
A B S T R A C T
Martensitically formed duplex fcc + hcp Co-based entropic alloys have been investigated both experimentally and theoretically. Theoretical predictions are in good agreement with experimental observations. A fair correlation is found between calculated driving forces for a partitionless $f c c \rightarrow h c p$ transformation and experimentally obtained phase fractions.
\end{abstract}

(C) 2019 Acta Materialia Inc. Published by Elsevier Ltd. This is an open access article under the CC BY license (http://creativecommons.org/licenses/by/4.0/).

\section{Background and motivation}

For economical and ethical reasons [1,2] as well as due to health issues [3-5] the possibility to substitute Cobalt with other elements has attracted a lot of attention in a wide range of different applications such as batteries, catalysts, superalloys and cemented carbides [6-11].

Cobalt-base alloys are used for corrosion and wear resistance and high temperature strength [12,13]. It is also an important alloying element in, for example, Ni-base superalloys [14,15] and high-speed steels $[16,17]$ and as the major component of the binder phase in cemented carbides [18,19].

Cobalt-base alloys and cobalt as an important alloying element in structural high temperature applications are favored for many reasons, for example, its fcc structure, the possibility to promote a duplex fcc + hcp structure and its low stacking fault energy [20-25]. For high temperature applications an fcc and/or a hcp

\footnotetext{
* Corresponding author. Unit of Structures, Dept Materials Science and Engineering, KTH, SE-10044, Stockholm, Sweden.

** Corresponding author.

E-mail addresses: ziyong@kth.se (Z. Hou), hlarsso@kth.se (H. Larsson).
}

lattice is beneficial compared to bcc because of the more sluggish diffusion in the former structures compared to the latter. Stacking faults are in effect layers of hcp structure embedded in an fcc matrix and the fewer number of glide systems in hcp compared to fcc hinder dislocation movement; stacking faults increase yield strength and reduce creep rate in temperature and stress regimes where creep mainly occur by dislocation movement [26]. By tailoring the relative fractions of fcc and hcp, and the stacking fault energy, through alloy design it is possible to vary ductility, hardness and creep properties in a wide range $[27,28]$.

In this work the possibility to partially substitute Cobalt with high-entropy alloys $[11,29,30]$ or, using the more appropriate term in the present context, so-called entropic alloys [31,32] has been investigated both theoretically and experimentally with a focus on the relative stabilities of the fcc and hcp structures. Moreover, for a long time high entropy alloys have been believed to form primarily bcc and/or fcc phases. The hcp phase has only been recently observed in CoCrFeMnNi [33], AlHfScTiZr [34], $\mathrm{Al}_{0.5} \mathrm{CoCrFeNi}$ [35] and $\mathrm{CoCrFeNi}$ [36]. Here we study derivatives of the CoCrFeMnNi system where the hcp and fcc phases co-exist.

The structure of this paper is as follows. In sections 2 and 3 the details of the experimental and theoretical work are given, 
respectively. In section 4 the alloy design is discussed with emphasis on thermodynamic calculations. Experimental results are compared with the theoretical predictions in section 5. Summary and conclusions are given in section 6 .

\section{Experimental details}

The alloys were prepared by arc melting high purity raw materials (purity $>99.5 \%$ ) in a pure Ar atmosphere. The samples were re-arc melted at least three times to reduce segregation.

Initial estimates for the homogenization heat treatment were performed by means of Scheil calculations using Thermo-Calc followed by Dictra simulations [37]. The Scheil calculations yield a conservative estimate of the micro segregation during casting. The segregation profiles were then used as input for Dictra simulations. The size of the domain was chosen as $100 \mu \mathrm{m}$ which roughly correspond to half the secondary dendrite arm spacing, rounded upwards. It was concluded that $175 \mathrm{~h}$ at $1473 \mathrm{~K}$ should be sufficient to ensure homogenization.

The heat treatment parameters from the calculations were shown to result in homogeneous samples by EDS measurements. During the heat treatment the alloy specimen were sealed in evacuated quartz tubes. After heat treatment the specimen were quenched to room temperature in water. One alloy was subsequently quenched in liquid nitrogen.

The homogenized and quenched samples were cut, ground, mechanically polished and finally electro-polished in an ethanol (90\%) - perchloric acid (10\%) solution for $30 \mathrm{~s}$ at $\sim 22 \mathrm{~V}$ followed by ultrasonic cleaning in ethanol.

Energy dispersive X-ray spectroscopy (EDS), electron channeling contrast imaging (ECCI) and electron backscatter diffraction (EBSD) were performed in a field emission scanning electron microscope JEOL JSM-7800F equipped with Bruker analytical systems EDS and EBSD detector. The accuracy of EDS used to determine the chemical composition of alloys is about $1 \%$. All the EDS measurements were taken with $20 \mathrm{kV}$ for $90 \mathrm{~s}$ to gain a sufficient signal. The average value from 25 analyzed points were taken for each alloy. EBSD measurements for phase identification and fraction analysis were taken at $15 \mathrm{kV}$ with a step size of $50 \mathrm{~nm}$.

X-ray diffraction (XRD) was performed using a Bruker D8 Discover X-ray Diffractometer in Bragg-Brentano geometry using $\mathrm{Cu}-\mathrm{K}_{\alpha}$ (wavelength $\lambda=1.5418 \AA$ ). The diffraction patterns were recorded from 35 to $90^{\circ} 2 \theta$ with angular steps of $0.015^{\circ}$ and a counting time of $2 \mathrm{~s}$ per step using a Lynxeye $1 \mathrm{D}$ detector at $40 \mathrm{kV}$ and $40 \mathrm{~mA}$. The background and the $\mathrm{Cu}-\mathrm{K}_{\alpha_{2}}$ signal were subtracted from the diffraction patterns prior to further analysis. The lattice constant for the samples were also derived from the measured XRD patterns. The peak positions were determined from Pseudo-Voigt fits to the measured diffractograms and converted into a lattice constant. The standard deviation for the peak position obtained in these fits was between $0.01^{\circ}$ and $0.05^{\circ}$ for the low and high diffraction angle regions, respectively.

Hardness was measured using a MXT CX-1 Matsuzawa microVickers indenter with a $50 \mathrm{~g}$ load (HV0.05) for $15 \mathrm{~s}$. The average hardness of each alloy was evaluated from 6 measurements.

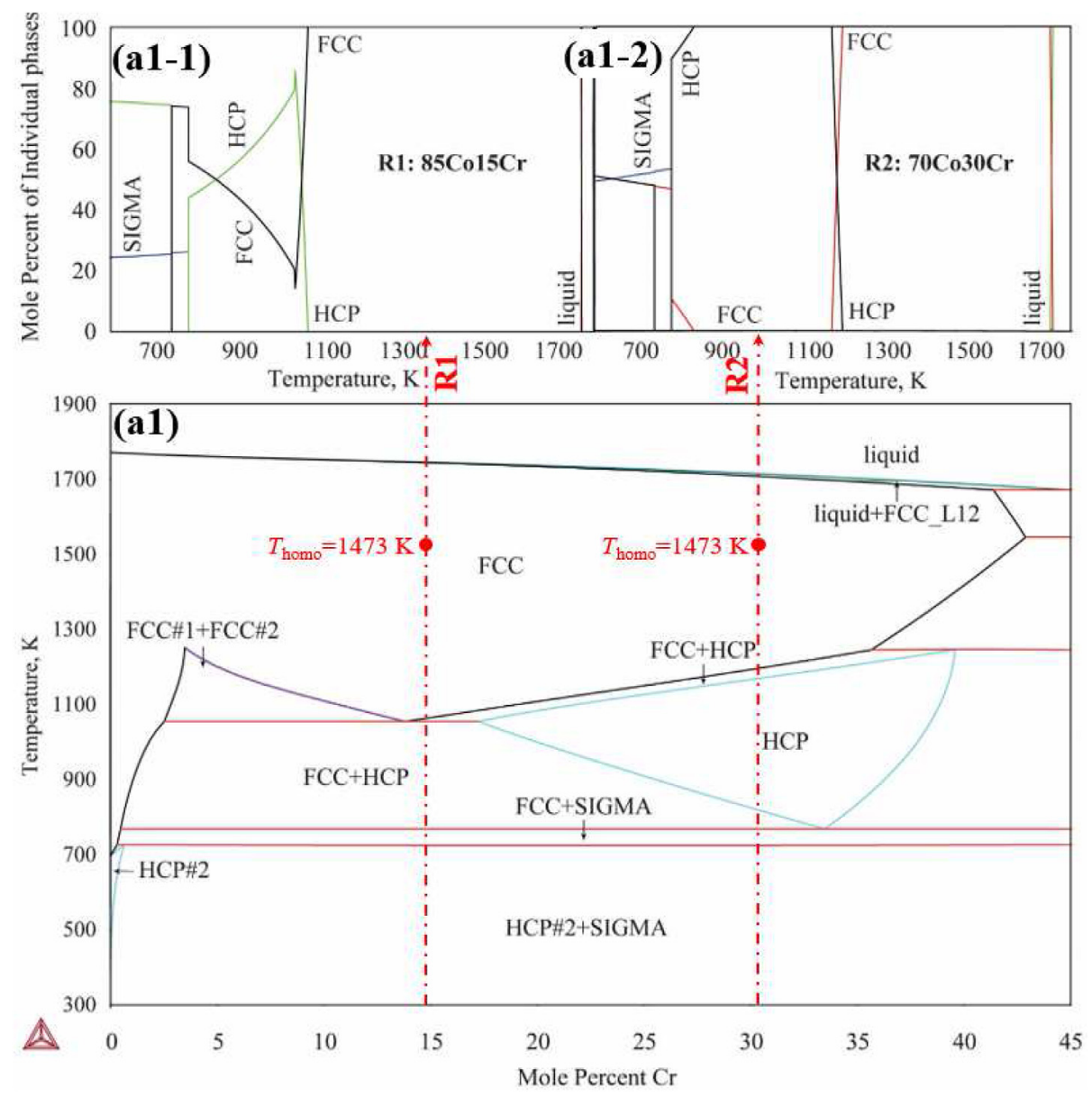

Fig. 1. The $\mathrm{Co}-\mathrm{Cr}$ phase diagram (bottom) and the phase fractions at equilibrium as a function of temperature for alloy R1 (top left) and R2 (top right). 
Table 1

Composition of alloys (at-\%) as obtained by EDS.

\begin{tabular}{lllllll}
\hline Alloy & Co & Cr & Fe & Mn & Ni & Nominal \\
\hline R1 & 85.27 & 14.71 & - & - & - & 85Co $15 \mathrm{Cr}$ \\
R2 & 70.55 & 29.41 & - & - & - & 70Co 30Cr \\
M1 & 72.06 & 14.43 & - & 6.80 & 7.53 & 70Co $15 \mathrm{Cr} 7.5 \mathrm{Mn} 7.5 \mathrm{Ni}$ \\
M2 & 56.75 & 29.45 & - & 6.76 & 7.59 & $55 \mathrm{Co} 30 \mathrm{Cr} 7.5 \mathrm{Mn} 7.5 \mathrm{Ni}$ \\
M3 & 71.57 & 14.52 & 7.41 & - & 7.56 & $70 \mathrm{Co} 15 \mathrm{Cr} 7.5 \mathrm{Fe} 7.5 \mathrm{Ni}$ \\
M4 & 56.11 & 29.61 & 7.44 & - & 7.62 & $55 \mathrm{Co} 30 \mathrm{Cr} 7.5 \mathrm{Fe} 7.5 \mathrm{Ni}$ \\
M5 & 63.96 & 14.37 & 7.37 & 6.78 & 7.51 & 62.5Co 15Cr 7.5Fe 7.5Mn 7.5Ni \\
M6 & 48.92 & 29.30 & 7.41 & 6.75 & 7.61 & $47.5 \mathrm{Co} 30 \mathrm{Cr} 7.5 \mathrm{Fe} 7.5 \mathrm{Mn} 7.5 \mathrm{Ni}$ \\
\hline
\end{tabular}

\section{Computational details}

The thermodynamic and kinetic calculations in this work were performed by the Thermo-Calc and Dictra softwares [37] using the thermodynamic database TCHEA2 $[38,39]$ and the kinetic database MOBNI4 [40].

Total energy calculations were performed in the framework of density functional theory (DFT) [41,42] as implemented in the exact muffin-tin orbital (EMTO) method [43-46]. EMTO is an improved screened Korringa-Kohn-Rostoker (KKR) method [47], where the exact one electron potential is represented by large overlapping muffin-tin potential spheres. By using overlapping spheres the exact crystal potential can be described more accurately when compared to the conventional muffin-tin or not overlapping approaches [44,48]. The Perdew-Burke-Ernzerhof generalized gradient approximation (PBE) was used for the exchange correlation functional [49]. A ferromagnetic state was assumed for all the calculations. In this study the chemical disorder in the random solid solutions was treated within the coherent-potential approximation (CPA) [50-55]. Nowadays, CPA is one of the most powerful techniques to treat random alloys due to the possibility to calculate properties of materials accurately using the unit cell instead of employing large and time consuming supercells [46]. Local relaxations can not be considered within the CPA approach, however, for the properties investigated in this study they appeared not to be significant as demonstrated earlier for CoCrFeNiW alloys $[11,56]$. The EMTO method together with CPA have been successfully applied to calculate the ground state properties of ordered [57,58] and disordered alloys [59] as well as HEA [60,61]. The full charge density (FCD) technique is implemented for accurate total energies [62,63]. The EMTO basis includes $s, p, d$ and $f$ orbitals. The one-electron equations were solved within the scalar relativistic approximation and the soft-core scheme [46]. The total number of k-points in the irreducible Brilloiun zone was 506, 916 and 1919 points for the bcc, fcc and hcp lattice. The screening parameter was kept constant throughout the calculations, 0.6020 .

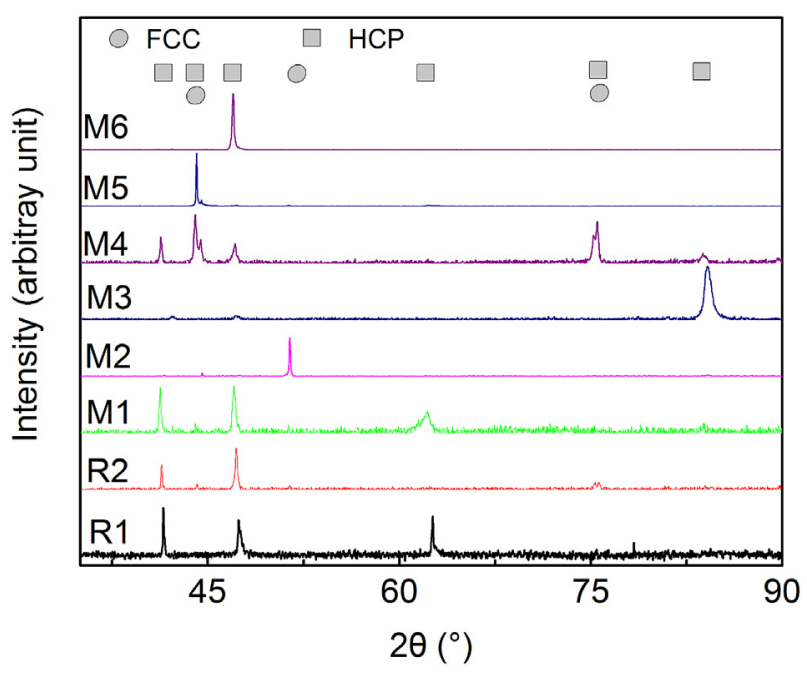

Fig. 3. XRD patterns of the alloys.

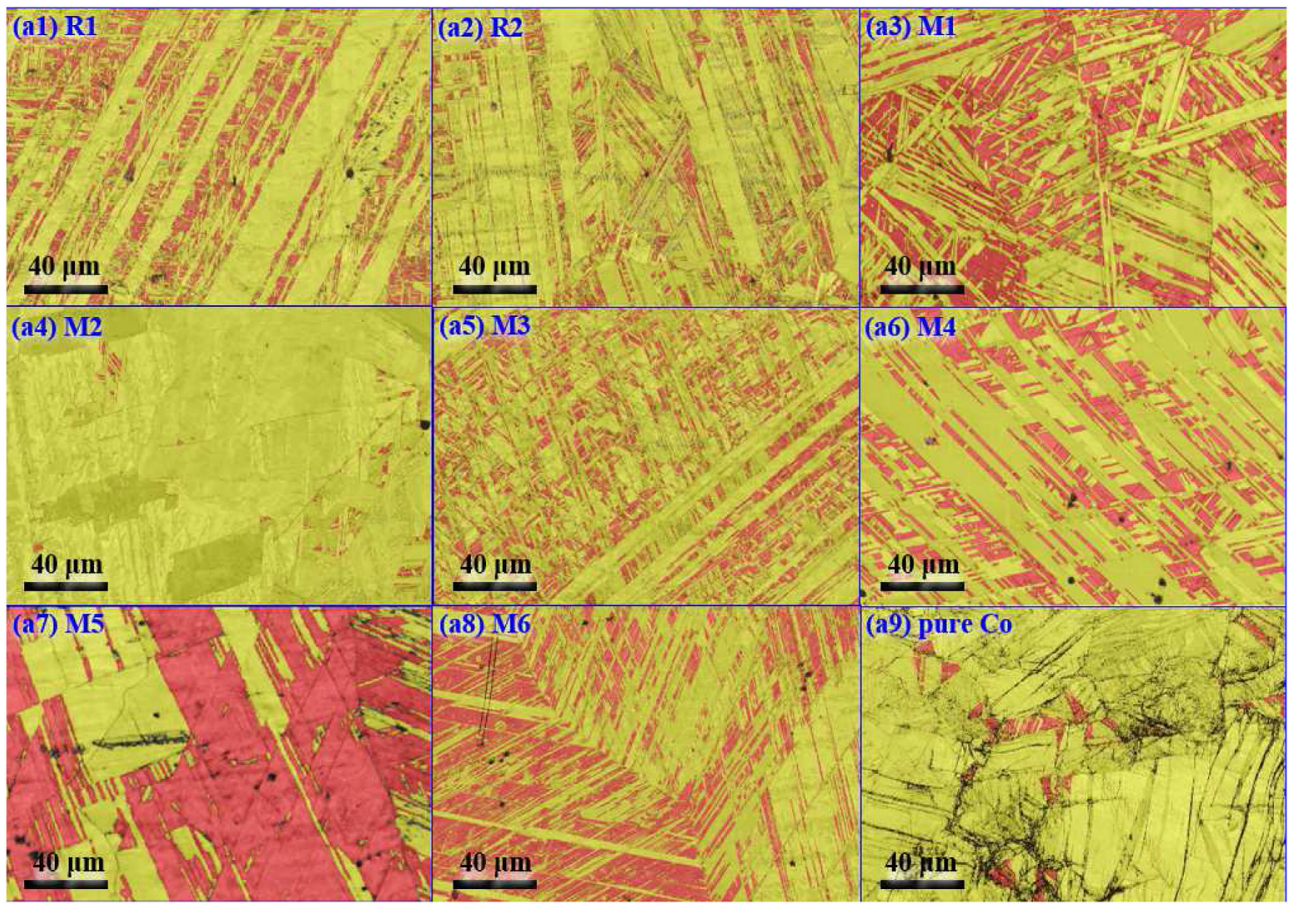

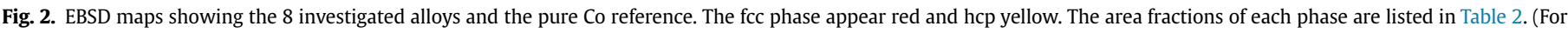
interpretation of the references to colour in this figure legend, the reader is referred to the Web version of this article.) 
Table 2

Various data for the alloys calculated using Thermo-Calc and measured area fraction of the hcp phase. Calculated quantities are in the 2 nd to 5 th columns. In the second column is listed the $T_{0}$ temperatures for fcc-hcp (i.e. the temperatures at which $G_{m}^{h c p}=G_{m}^{f c c}$ ), in the third and fourth columns are the driving forces $D_{m}$ for partitionless transformations, the fifth column contain the atomic $f c c \rightarrow h c p$ volume change $\Delta V . D_{m}$ and $\Delta V$ were calculated at $300 \mathrm{~K}$. The experimentally measured area fraction hcp is listed in the sixth column. The area fraction hcp in the quenched pure Co reference is $94.8 \%$.

\begin{tabular}{|c|c|c|c|c|c|}
\hline \multirow[t]{2}{*}{ Alloy } & \multirow{2}{*}{$\begin{array}{l}\frac{T_{0}[\mathrm{~K}]}{f c c-} \\
h c p\end{array}$} & \multicolumn{2}{|c|}{$D_{m}[\mathrm{~J} / \mathrm{mol}]$} & \multirow{2}{*}{$\frac{\Delta V\left[\AA^{3}\right]}{f c c \rightarrow h c p}$} & \multirow{2}{*}{$\frac{\% \text { hcp }}{\text { (bal. fcc }}$} \\
\hline & & $f c c \rightarrow b c c$ & $f c c \rightarrow h c p$ & & \\
\hline $\mathrm{R} 1$ & 1044 & -677 & 1286 & -0.16 & 82.1 \\
\hline $\mathrm{R} 2$ & 1173 & -856 & 2101 & -0.29 & 88.6 \\
\hline M1 & 915 & -356 & 854 & -0.21 & 66.8 \\
\hline M2 & 1094 & 538 & 1569 & -0.28 & 99.0 \\
\hline M3 & 563 & -1294 & 535 & -0.16 & 80.1 \\
\hline M4 & 849 & -1379 & 1382 & -0.26 & 75.3 \\
\hline M5 & 559 & -1404 & 471 & -0.22 & 37.6 \\
\hline M6 & 834 & -593 & 1143 & -0.30 & 66.1 \\
\hline
\end{tabular}

\section{Design of alloys}

The alloys were designed to facilitate the investigation on the influence of different alloying elements on the relative stabilities of fcc and hcp. Cr was chosen as the major alloying element. Chromium will generally improve corrosion resistance, but in the present context the presence of the large hcp single phase region at fairly high $\mathrm{Cr}$ contents was of equal or higher importance. The $\mathrm{Co}-\mathrm{Cr}$ phase diagram is shown in Fig. 1. A prerequisite for all alloys were that they should consist solely of fcc at the homogenizing temperature $(1473 \mathrm{~K})$ to mimic pure Co. Furthermore, at the temperature directly below this one there should be a correspondingly large solid solubility range for the hcp phase. Such a feature of phase relations facilitates the martensitic transformation from fcc to hcp during quenching. The nominal and measured compositions of alloys are given in Table 1 . Two reference binary $\mathrm{Co}-\mathrm{Cr}$ alloys were made with approximately 15 and 30 at-\% Cr, respectively.

\section{Results}

EBSD maps of all studied alloys showing the structure after quenching from the homogenization temperature are shown in Fig. 2. For reference, an EBSD map of pure Co is also included. Only fcc and hcp were detected and this was further validated by XRD, see Fig. 3. The phase fractions included in Table 2 were obtained from these maps except for alloy M5 for which an additional scan of a larger area was made due to the coarseness of its microstructure. The area fraction of hcp in the quenched pure Co reference is $94.8 \%$. The homogeneity of the alloys were checked by EDS line-scans, which also show that the $f c c \rightarrow h c p$ transformation is partitionless, as expected. Fig. 4 shows the result of a line scan in alloy M6. The hardness of the alloys in the quenched state is shown in Fig. 5. Fig. 6 shows XRD pattern and EBSD maps of alloy M5 after quenching in liquid nitrogen, which resulted in the formation of an additional $\sim$ $27 \%$ of hcp.

Results from thermodynamic and DFT calculations are summarized in Tables 2 and 3, respectively. The total energies as a function of volume as obtained from DFT calculations are shown in Fig. 7.

According to both thermodynamic (calculated at $300 \mathrm{~K}$ ) and DFT calculations hcp is the most stable solid solution phase for all alloys. According to the thermodynamic calculations there is a positive driving force for an $f c c \rightarrow b c c$ transformation for alloy $\mathrm{M} 2$, but smaller than the corresponding driving force for $f c c \rightarrow h c p$. Experimentally, only fcc and hcp are observed. Further, it is expected that the $f c c \rightarrow h c p$ martensitic transformation require less driving force than $f c c \rightarrow b c c$ due to the smaller volume change [66,67]. There is thus a good qualitative agreement between calculations and experimental observations.

The energy difference between fcc and hcp as calculated by DFT is very small, less than $3.25 \mathrm{~kJ} / \mathrm{mol}$ for all the alloys. In particular, M1, M2, M3, M4 and M6 closely resemble pure Co, where this energy difference is of the order of $2.3 \mathrm{~kJ} / \mathrm{mol}$. R1 and R2 have a little higher energy difference between the hcp and fcc phases. The bcc phase is energetically higher than fcc and hcp for all the cases, consistent with the fact that the phase is not observed

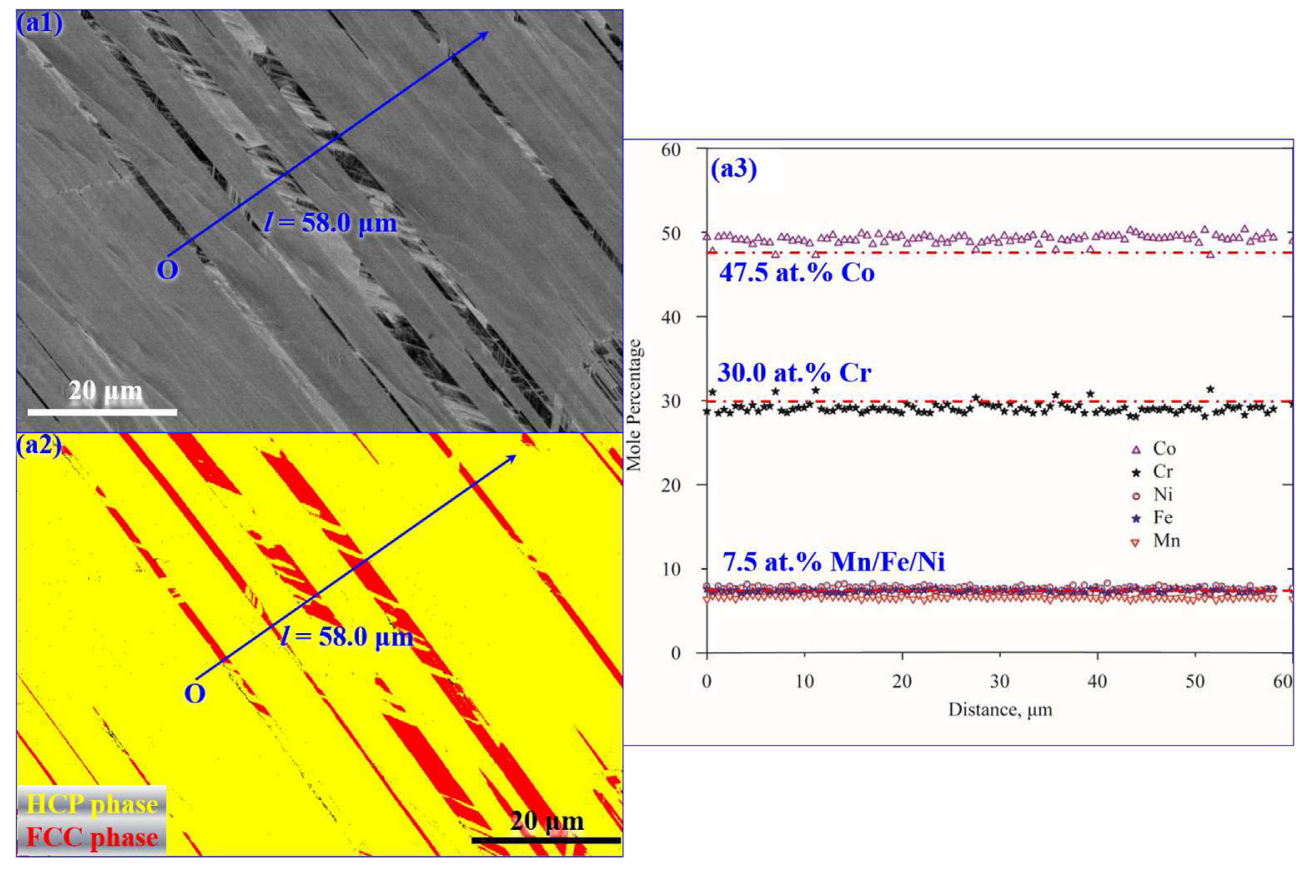

Fig. 4. ECCI image (a1) and corresponding phase map (a2) of Alloy M6. Line-scan EDS (a3) show the homogenity of the alloy. 


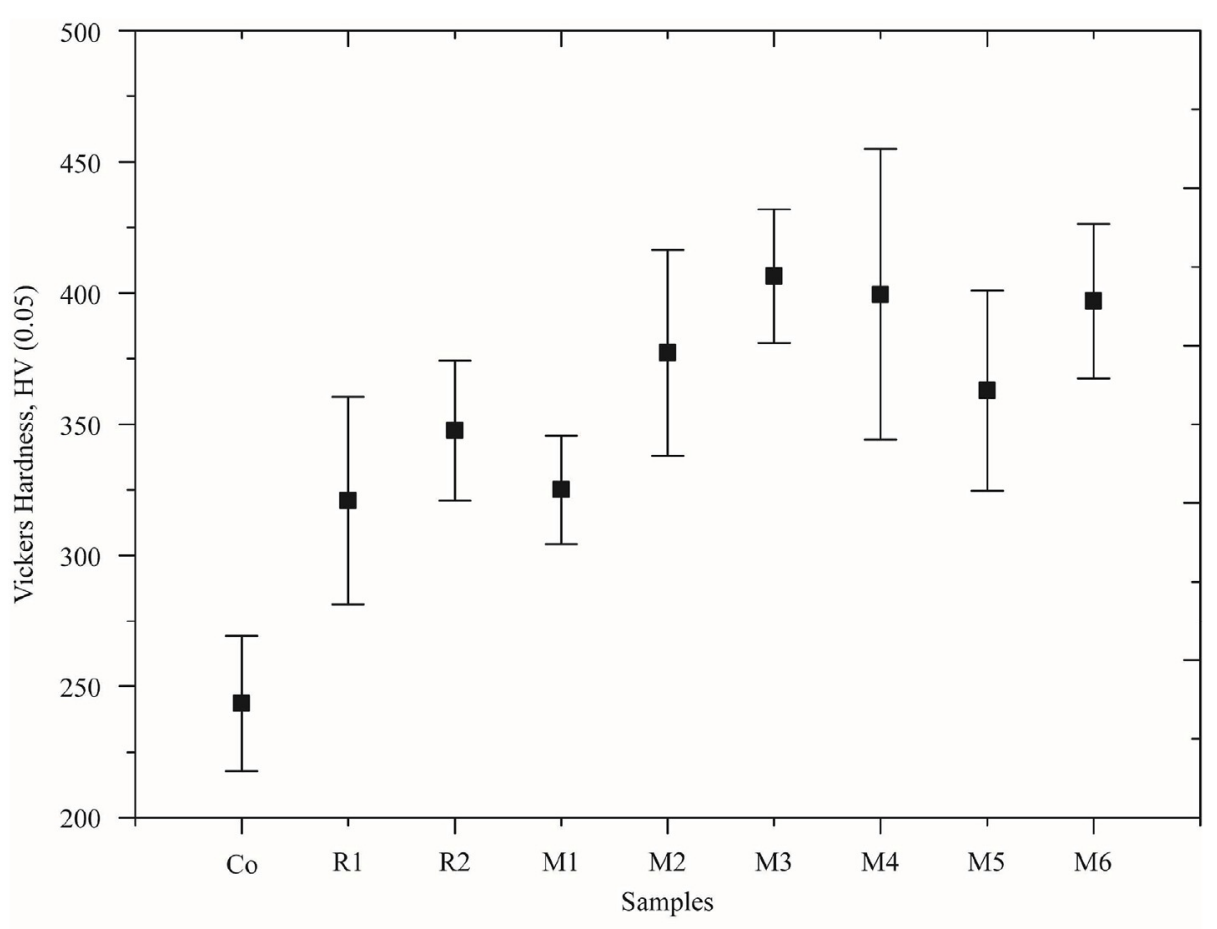

Fig. 5. The Vickers hardness of the alloys and the pure Co reference.

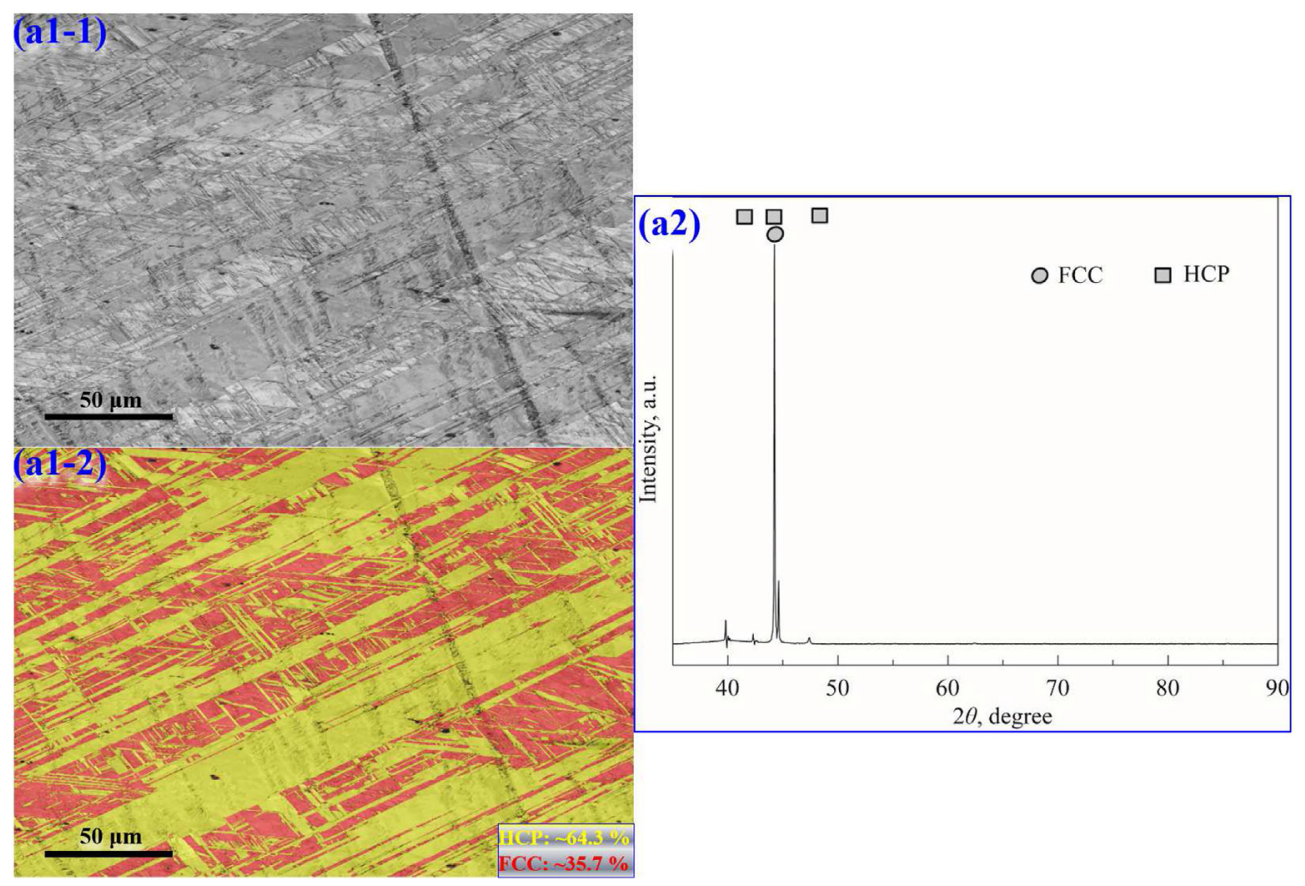

Fig. 6. ECCI (a1-1) with EBSD phase map (a1-2) and XRD pattern (a2) of alloy M5 after quenching in liquid nitrogen. 
Table 3

Total energy differences, $\Delta E_{b c c-h c p}$ and $\Delta E_{f c c-h c p}$, as obtained from DFT calculations. The calculated and experimentally determined lattice constants $a$ for bcc, fcc and hcp phases and c/a are listed as well. Experimental lattice parameters for pure Co were taken from Ref. [64]. DFT calculated values of the energy difference for pure Co is taken from Ref. [65].

\begin{tabular}{|c|c|c|c|c|c|c|c|c|c|}
\hline \multirow[t]{3}{*}{ Alloy } & \multicolumn{2}{|c|}{$\Delta \mathrm{E}(\mathrm{kJ} / \mathrm{mol})$} & \multicolumn{5}{|l|}{ a $(\AA)$} & \multirow{2}{*}{\multicolumn{2}{|c|}{$\frac{\mathrm{c} / \mathrm{a}}{\mathrm{hcp}}$}} \\
\hline & \multirow[t]{2}{*}{ bcc-hcp } & \multirow[t]{2}{*}{ fcc-hcp } & \multirow[t]{2}{*}{ bcc } & \multicolumn{2}{|l|}{ fcc } & \multicolumn{2}{|l|}{ hcp } & & \\
\hline & & & & theo. & exp. & theo. & exp. & theo. & exp. \\
\hline Co & - & 2.3 & - & 3.53 & 3.5446 & 2.50 & 2.5071 & 1.62 & 1.623 \\
\hline R1 & 8.38 & 3.25 & 2.82 & 3.52 & 3.55 & 2.50 & 2.51 & 1.61 & 1.62 \\
\hline $\mathrm{R} 2$ & 3.75 & 2.69 & 2.82 & 3.52 & 3.55 & 2.50 & 2.52 & 1.61 & 1.61 \\
\hline M1 & 6.17 & 1.90 & 2.82 & 3.53 & 3.56 & 2.50 & 2.53 & 1.61 & 1.63 \\
\hline M2 & 4.99 & 2.11 & 2.82 & 3.52 & 3.52 & 2.49 & 2.51 & 1.61 & 1.62 \\
\hline M3 & 6.68 & 2.23 & 2.82 & 3.54 & 3.53 & 2.51 & 2.51 & 1.61 & 1.63 \\
\hline M4 & 4.80 & 1.97 & 2.82 & 3.53 & 3.54 & 2.50 & 2.52 & 1.61 & 1.64 \\
\hline M5 & 4.37 & 1.59 & 2.83 & 3.54 & 3.54 & 2.50 & 2.50 & 1.62 & 1.64 \\
\hline M6 & 3.48 & 2.16 & 2.83 & 3.53 & 3.50 & 2.49 & 2.53 & 1.61 & 1.62 \\
\hline
\end{tabular}

experimentally. The calculated lattice constants for bcc, fcc and hcp are displayed in Table 3. For comparison the same values as obtained from the XRD data are also listed. We observe excellent agreement between experiments and calculations with a deviation no larger than $0.8 \%$ for a and $1.8 \%$ for $\mathrm{c} / \mathrm{a}$.

Attempts were made to correlate the measured area fractions of hcp and fcc with data obtained from calculations. However, it is clear that for a sophisticated model more data is needed. Nevertheless, there is a reasonable correlation between the driving forces obtained from thermodynamic calculations and the measured phase area fractions. Using a simple JMAK (Johnson-Mehl-Avrami-Kolmogorov) [68-72] type of expression, viz.

$f_{h c p}=1-\exp \left[k\left(\frac{D_{m}}{R T}\right)^{n}\right]$

a reasonable fit with experimental data was obtained, see Fig. 8 which show results obtained using data obtained from ThermoCalc. In Eq. (1) $k$ and $n$ are fitting parameters, $D_{m}$ is the calculated

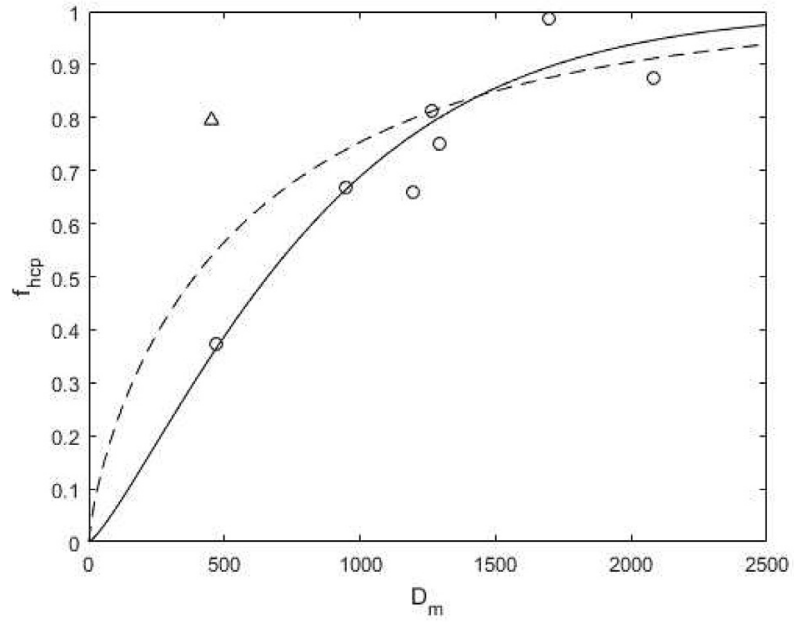

Fig. 8. Two least squares fits of experimental data on fraction hcp as a function of driving force using Eq. (1). For the solid line the experimental point given by the triangle was excluded from the fitting procedure.

driving force and $f_{\text {hcp }}$ is the measured area fraction of hcp. A much improved fit was obtained by excluding the "outlier" corresponding to alloy M3 (shown as a triangular experimental point in Fig. 8), which yielded $k=-3.66$ and $n=1.25$.

\section{Summary and conclusions}

A number of different Co-based entropic alloys were prepared and investigated experimentally. After homogenizing at $1473 \mathrm{~K}$ and quenching to room temperature only the fcc and hcp phases were detected by EBSD and XRD, in agreement with theoretical predictions. The phase fractions correlate reasonably well with thermodynamic calculations of the driving forces for partitionless transformations; A simple JMAK model give a fair fit, though it should be stressed that this fit is not expected to hold outside the experimentally investigated composition range.

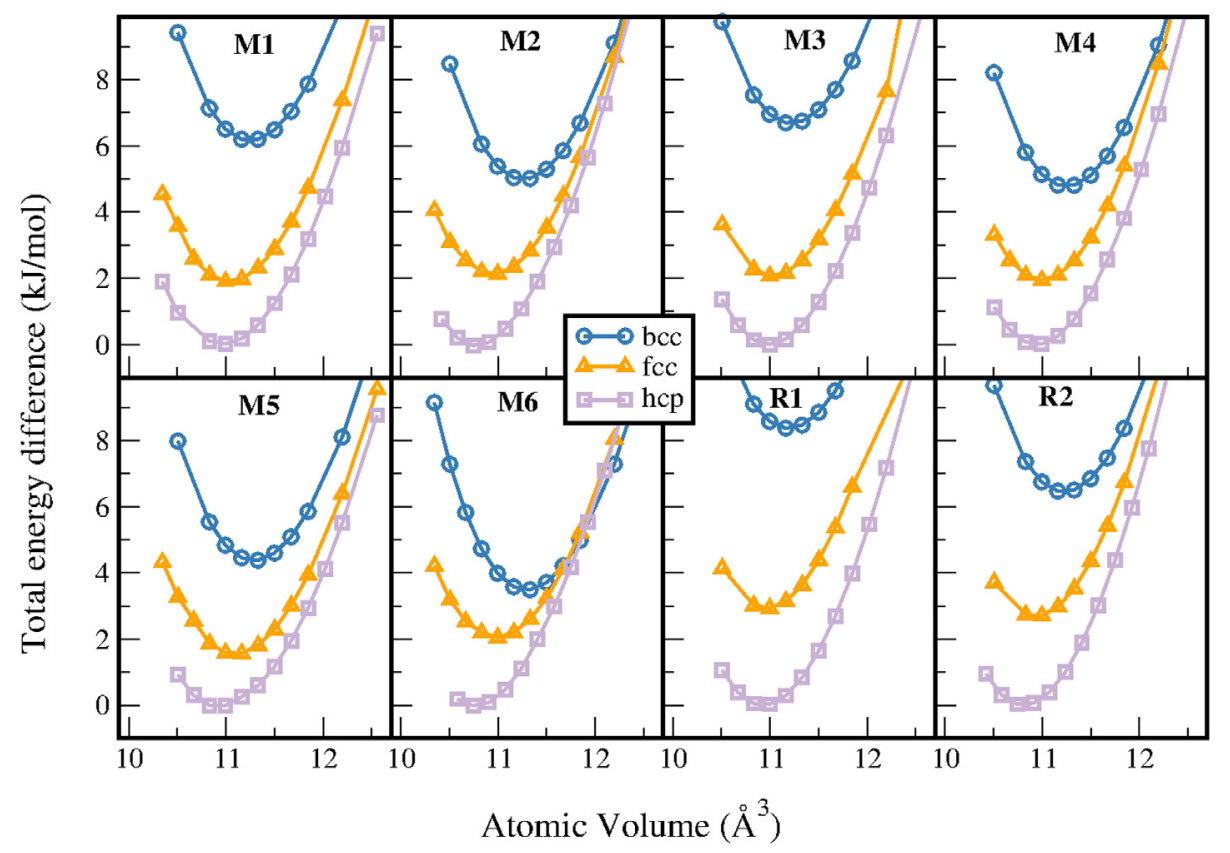

Fig. 7. Results from DFT calculations. 
The present work show the feasibility of computational tools for designing duplex fcc + hcp Co-based entropic alloys. However, a more sophisticated model for the martensitic $f c c \rightarrow h c p$ transformation would be highly useful, but more experimental data is needed for the development of such a model.

\section{Acknowledgement}

Funding from Vinnova, Sweden's Innovation Agency, grant 2016-00805, is gratefully acknowledged.

\section{References}

[1] M.L. Grilli, T. Bellezze, E. Gamsjäger, A. Rinaldi, P. Novak, S. Balos, R.R. Pitiescu, M.L. Ruello, Solutions for critical raw materials under extreme conditions: a review, Materials 10 (2017) 285.

[2] E. Airhart, Alternatives to Cobalt, the Blood Diamond of Batteries, Wired Magazine, June 72018.

[3] B. Nemery, J.L. Abraham, Hard metal lung disease: still hard to understand, Am. J. Respir. Crit. Care Med. 176 (2007) 2-3.

[4] National Institute of Health, Tech. Rep. 14-5923, National Toxicology Program, U.S. Department of Health and Human Services, 2013.

[5] A. Linna, P. Oksa, K. Groundstroem, M. Halkosaari, P. Palmroos, S. Huikko, J. Uitti, Exposure to cobalt in the production of cobalt and cobalt compounds and its effect on the heart, Occup. Environ. Med. 61 (2004) 877-885.

[6] J. Lee, D.A. Kitchaev, D.-H. Kwon, C.-W. Lee, J.K. Papp, Y.-S. Liu, Z. Lun, R.J. Clément, T. Shi, B.D. McCloskey, J. Guo, M. Balasubramanian, G. Ceder, Reversible Mn2+/Mn4+ double redox in lithium-excess cathode materials Nature 556 (2018) 185-190.

[7] F. Kallmeier, R. Kempe, Manganese complexes for (De)Hydrogenation catalysis: a comparison to cobalt and iron catalysts, Angew. Chem. Int. Ed. 57 (2018) 46-60.

[8] J.K. Tien, T.E. Howson, G.L. Chen, X.S. Xie, Cobalt availability and superalloys, Journal of Metals 32 (1980) 12-20.

[9] C.M. Fernandes, A.M.R. Senos, Cemented carbide phase diagrams: a review, Int. J. Refract. Met. H. 29 (2011) 405-418.

[10] O.J. Ojo-Kupoluyi, S.M. Tahir, B.T.H.T. Baharudin, M.A. Azmah Hanim, M.S. Anuar, Mechanical properties of WC-based hardmetals bonded with iron alloys a review, Mater. Sci. Tech.-Lond. 33 (2017) 507-517.

[11] E. Holmström, R. Lizárraga, D. Linder, A. Salmasi, W. Wang, B. Kaplan, H. Mao, H. Larsson, L. Vitos, High entropy alloys: substituting for cobalt in cutting edge technology, Appl. Mater. Today 12 (2018) 322-329.

[12] D.L. Klarstrom, Wrought cobalt- base superalloys, J. Mater. Eng. Perform. 2 (1993) 523-530.

[13] D. Coutsouradis, A. Davin, M. Lamberigts, Cobalt-based superalloys for applications in gas turbines, Mater. Sci. Eng. 88 (1987) 11-19.

[14] R.C. Reed, The Superalloys - Fundamentals and Applications, Cambridge University Press, Cambridge, 2006.

[15] T.M. Pollock, S. Tin, Nickel-based superalloys for advanced turbine engines: chemistry, microstructure and properties, J. Propuls. Power 22 (2006) $361-374$.

[16] H.F. Fischmeister, S. Karagöz, H.-O. Andrén, An atom probe study of secondary hardening in high speed steels, Acta Metall. 36 (1988) 817-825.

[17] W. Rong, H.-O. Andrén, H. Wisell, G.L. Dunlop, The role of alloy composition in the precipitation behaviour of high speed steels, Acta Metall. Mater. 40 (1992) 1727-1738.

[18] H.E. Exner, Physical and chemical nature of cemented carbides, Int. Met. Rev. 24 (1979) 149-173.

[19] X. Ren, H. Miao, Z. Peng, A review of cemented carbides for rock drilling: an old but still tough challenge in geo-engineering, Int. J. Refract. Met. H. 39 (2013) 61-77.

[20] H.M. Tawancy, V.R. Ishwar, B.E. Lewis, On the fcc $\rightarrow$ hcp transformation in a cobalt-base superalloy (Haynes alloy No. 25), J. Mater. Sci. Lett. 5 (1986) $337-341$.

[21] L.-Y. Tian, R. Lizárraga, H. Larsson, E. Holmström, L. Vitos, A first principles study of the stacking fault energies for fcc Co-based binary alloys, Acta Mater 136 (2017) 215-223.

[22] X. Wu, N. Tao, Y. Hong, J. Lu, K. Lu, $\gamma \rightarrow \varepsilon$ martensite transformation and twinning deformation in fcc cobalt during surface mechanical attrition treatment, Scripta Mater. 52 (2005) 547-551.

[23] P. Tolédano, G. Krexner, M. Prem, H.-P. Weber, V.P. Dmitriev, Theory of the martensitic transformation in cobalt, Phys. Rev. B 64 (2001) 144104

[24] C.K.L. Davies, P.W. Davies, B. Wilshire, The effect of variations in stacking-fault energy on the creep of nickel-cobalt alloys, Philos. Mag. 12 (1965) 827-839.

[25] R.N. Jarrett, L. Chin, J.K. Tien, The Role of Cobalt on the Creep of Waspaloy, NASA Technical Report, NASA-CR-174628, NAS 1.26:174628, NASA, 1984.

[26] G.E. Dieter, Mechanical Metallurgy, McGraw-Hill, London, 1988.

[27] L. Remy, A. Pineau, Twinning and strain-induced F.C.C. $\rightarrow$ H.C.P. transformation in the Fe-Mn-Cr-C system, Mater. Sci. Eng. 28 (1977) 99-107.

[28] B.C. De Cooman, Y. Estrin, S.K. Kim, Twinning-induced plasticity (TWIP) steels, Acta Mater. 142 (2018) 283-362.
[29] J.-W. Yeh, S.-K. Chen, S.-J. Lin, J.-Y. Gan, T.-S. Chin, T.-T. Shun, C.-H. Tsau, S.Y. Chang, Nanostructured high-entropy alloys with multiple principal elements: novel alloy design concepts and outcomes, Adv. Eng. Mater. 6 (2004) 299-303.

[30] B.S. Murty, J.-W. Yeh, S. Ranganathan, High-Entropy Alloys, Elsevier Science, Amsterdam, 2014

[31] L. Shao, T. Zhang, L. Li, Y. Zhao, J. Huang, P.K. Liaw, Y. Zhang, A low-cost lightweight entropic alloy with high strength, J. Mater. Eng. Perform. 27 (2018) 6648-6656.

[32] S. Xia, C.M. Lousada, H. Mao, A.C. Maler, P.A. Korzhavyi, R. Sandström, Y. Wang, Y. Zhang, Nonlinear oxidation behavior in pure Ni and Ni-containing entropic alloys, Front. Mater. 5 (2018) 53.

[33] C.L. Tracy, P. Park, D.R. Rittman, S.J. Zinkle, H. Bei, M. Lang, R.C. Ewing, W.L. Mao, High pressure synthesis of a hexagonal close-packed phase of the high-entropy alloy CrMnFeCoNi, Nat. Commun. 8 (2017) 15634.

[34] L. Rogal, P. Bobrowski, F. Körmann, S. Divinski, F. Stein, B. Grabowski, Computationally-driven engineering of sublattice ordering in a hexagonal AlHfScTiZr high entropy alloy, Sci. Rep. 7 (2017) 2209.

[35] J. Wang, Y. Zhang, S.Z. Niu, W.Y. Wang, H.C. Kou, J.S. Li, Formation of a hexagonal closed-packed phase in Al0.5CoCrFeNi high entropy alloy, MRS Commun 7 (2017) 879-884.

[36] F.X. Zhang, S. Zhao, K. Jin, H. Bei, D. Popov, C. Park, J.C. Neuefeind, W.J. Weber, Y. Zhang, Pressure-induced fcc to hcp phase transition in Ni-based high entropy solid solution alloys, Appl. Phys. Lett. 110 (2017), 011902.

[37] J.-O. Andersson, T. Helander, L. Höglund, P. Shi, B. Sundman, Thermo-Calc \& DICTRA, computational tools for materials science, Calphad 26 (2002) $273-312$.

[38] H. Chen, H. Mao, Q. Chen, Database development and Calphad calculations for high entropy alloys: challenges, strategies, and tips, Mater. Chem. Phys. 210 (2018) 279-290.

[39] H. Mao, H. Chen, Q. Chen, TCHEA1: a thermodynamic database not limited for high entropy alloys, J. Phase Equilibria Diffusion 38 (2017) 353-368.

[40] Thermo-Calc Software, www.thermocalc.com

[41] P. Hohenberg, W. Kohn, Inhomogeneous electron gas, Phys. Rev. 136 (1964) B864-B871.

[42] W. Kohn, L.J. Sham, Self-consistent equations including exchange and correlation effects, Phys. Rev. 140 (1965) A1133-A1138.

[43] L. Vitos, H.L. Skriver, B. Johansson, J. Kollár, Application of the exact muffin-tin orbitals theory: the spherical cell approximation, Comput. Mater. Sci. 18 (2000) 24-38.

[44] L. Vitos, Total-energy method based on the exact muffin-tin orbitals theory, Phys. Rev. B 64 (2001), 014107.

[45] L. Vitos, I.A. Abrikosov, B. Johansson, Anisotropic lattice distortions in random alloys from first-principles theory, Phys. Rev. Lett. 87 (2001) 156401.

[46] L. Vitos, Computational Quantum Mechanics for Materials Engineers: the EMTO Method and Applications, Springer-Verlag, London, 2007.

[47] O.K. Andersen, O. Jepsen, G. Krier, Exact muffin-tin orbital theory, in: V. Kumar, O.K. Andersen, A. Mokerjee (Eds.), Lectures on Methods of Electronic Structure Calculations, World Scientific, Singapore, 1994, pp. 63-124.

[48] O.K. Andersen, C. Arcangeli, R.W. Tank, T. Saha-Dasgupta, G. Krier, O. Jepsen, I. Dasgupta, Third-generation TB-LMTO, MRS Proc 491 (1997) 3.

[49] J.P. Perdew, K. Burke, M. Ernzerhof, Generalized gradient approximation made simple, Phys. Rev. Lett. 77 (1996) 3865-3868.

[50] P. Soven, Coherent-potential model of substitutional disordered alloys, Phys. Rev. 156 (1967) 809-813.

[51] B.L. Gyorffy, Coherent-potential approximation for a nonoverlapping-muffintin-potential model of random substitutional alloys, Phys. Rev. B 5 (1972) 2382-2384.

[52] D.W. Taylor, Vibrational properties of imperfect crystals with large defect concentrations, Phys. Rev. 156 (1967) 1017-1029.

[53] D.D. Johnson, D.M. Nicholson, F.J. Pinski, B.L. Gyorffy, G.M. Stocks, Densityfunctional theory for random alloys: total energy within the coherentpotential approximation, Phys. Rev. Lett. 56 (1986) 2088.

[54] D.D. Johnson, D.M. Nicholson, F.J. Pinski, B.L. Gyorffy, G.M. Stocks, Total-energy and pressure calculations for random substitutional alloys, Phys. Rev. B 41 (1990) 9701.

[55] D.D. Johnson, F.J. Pinski, Inclusion of charge correlations in calculations of the energetics and electronic structure for random substitutional alloys, Phys. Rev. B 48 (1993) 11553.

[56] R. Lizárraga, E. Holmström, L. Vitos, Alloying effect of tungsten on the structural and magnetic properties of CoCrFeNiW high entropy alloys, Phys. Rev. Mater. 2 (2018), 094407.

[57] Z.W. Lu, S.H. Wei, A. Zunger, Electronic structure of ordered and disordered $\mathrm{Cu}_{3} \mathrm{Au}$ and , $\mathrm{Cu}_{3}$ Pd, Phys. Rev. B 45 (1992) 10314-10330.

[58] G.-S. Wang, E.K. Delczeg-Czirjak, Q.-M. Hu, K. Kokko, B. Johansson, L. Vitos, The effect of long-range order on the elastic properties of $\mathrm{Cu}_{3} \mathrm{Au}$, J. Phys. Condens. Matter 25 (2013), 085401.

[59] L.-Y. Tian, Q.-M. Hu, R. Yang, J. Zhao, B. Johansson, L. Vitos, Elastic constants of random solid solutions by SQS and CPA approaches: the case of fcc Ti-Al, J. Phys. Condens. Matter 27 (2015) 315702.

[60] S. Huang, A. Vida, D. Molnár, K. Kádas, L.K. Varga, E. Holmström, L. Vitos, Phase stability and magnetic behavior of FeCrCoNiGe high-entropy alloy, Appl. Phys. Lett. 107 (2015) 251906.

[61] S. Huang, W. Li, X. Li, S. Schönecker, L. Bergqvist, E. Holmström, L.K. Varga, L. Vitos, Mechanism of magnetic transition in FeCrCoNi-based high entropy 
alloys, Mater. Des. 103 (2016) 71-74.

[62] J. Kollár, L. Vitos, H.L. Skriver, in: H. Dreysse (Ed.), Electronic Structure and Physical Properties of Solids, Springer, London, 2000, pp. 85-114.

[63] L. Vitos, J. Kollár, H. Skriver, Full charge-density scheme with a kinetic-energy correction: application to ground-state properties of the $4 \mathrm{~d}$ metals, Phys. Rev. B 55 (1997) 13521-13527.

[64] T. Nishizawa, K. Ishida, The Co (Cobalt) system, Bull. Alloy Ph. Diag. 4 (1983) 387-390.

[65] R. Lizárraga, F. Pan, L. Bergqvist, E. Holmström, Z. Gercsi, L. Vitos, First principles theory of the hcp-fcc phase transition in cobalt, Sci. Rep. 7 (2017) 3778.

[66] A. Borgenstam, Nucleation and Growth of Martensite in Steel, Thesis, KTH, Stockholm, Sweden, 1997.
[67] M. Andersson, Thermal and Stress Induced $\gamma \rightarrow \varepsilon$ Martensitic Transformations in Fe-Mn-Si Shape Memory Alloys, thesis, KTH, Stockholm, Sweden, 1997.

[68] A. Kolmogoroff, Zur statistik der Kristallisationsvorgänge in metallen, Izv. Akad. Nauk SSSR Ser. Mat. 1 (1937) 355-359.

[69] W.A. Johnson, R.F. Mehl, Reaction kinetics in processes of nucleation and growth, Trans. AIME 135 (1939) 416-458.

[70] M. Avrami, Kinetics of phase change. I general theory, J. Chem. Phys. 7 (1939) 1103-1142.

[71] M. Avrami, Kinetics of phase change. II TransformationTime relations for random distribution of nuclei, J. Chem. Phys. 8 (1940) 212-224.

[72] M. Avrami, Granulation, phase change, and microstructure kinetics of phase change. III, J. Chem. Phys. 9 (1941) 177-184. 